\title{
Tissue-Specific Fc $\gamma$ and Complement Receptor Expression by Alveolar Macrophages Determines Relative Importance of IgG and Complement in Promoting Phagocytosis of Pseudomonas aeruginosa
}

\author{
MELVIN BERGER, TONYA M. NORVELL, MICHAEL F. TOSI, STEVEN N. EMANCIPATOR, \\ MICHAEL W. KONSTAN, AND JOHN R. SCHREIBER \\ Departments of Pediatrics and Pathology, Case Western Reserve University School of Medicine. \\ Cleveland, Ohio 44102
}

\begin{abstract}
Because the expression of IgG Fc receptors and complement receptors on macrophages may vary in a tissue-specific manner, we used monoclonal antibodies and flow cytometry to define the expression and function of opsonin receptors on fresh normal and cystic fibrosis (CF) bronchoalveolar lavage (BAL) macrophages. Using flow cytometry to separately analyze individual types of cells, we then determined the relative contributions of IgG and complement to phagocytosis of Pseudomonas aeruginosa by fresh BAL cells, avoiding alterations in receptor expression due to in vitro purification or culturing techniques. Neither normal nor CF BAL macrophages express appreciable amounts of the complement receptors CR1, CR2, or CR3. These results were confirmed by immunohistochemical staining of fixed lung sections. BAL macrophages express a high-affinity IgG receptor, Fc $\gamma R I$, that is not found on neutrophils (PMN). In contrast, chemoattractantstimulated blood PMN express large amounts of CR1 and CR3 but do not express Fc $\gamma$ RI. These results correlate with phagocytosis assays, which show that phagocytosis by macrophages is enhanced by relatively low concentrations of IgG but that the addition of complement does not further increase their phagocytosis. In contrast, low concentrations of IgG alone do not promote phagocytosis by PMN, whereas addition of complement markedly enhances phagocytosis by PMN. These results may explain the previously reported sensitivity of macrophages rather than PMN to the "blocking" effects of anti-Pseudomonas antibodies from CF patients, and emphasize the pathologic significance of interference with IgG and complement mediated opsonization in the lung in CF. (Pediatr Res 35: 6877, 1994)
\end{abstract}

\section{Abbreviations}

AM, alveolar macrophage

BAL, bronchoalveolar lavage

CD, cluster of differentiation

CF, cystic fibrosis

CR, complement receptor

Fc $\gamma R$, receptor for the Fc portion of $\mathrm{IgG}$

FEV $V_{1}$, forced expiratory volume in $1 \mathrm{~s}$

FMLP, $\boldsymbol{N}$-formyl-methionylleucylphenylalanine

Received April 2, 1993; accepted August 12, 1993.

Correspondence: Dr. Melvin Berger, Rainbow Babies and Childrens Hospital

2101 Adelbert Road, Room 594, Cleveland, OH 44106.

Supported by National Institutes of Health grants DK 27651, AI 27862, HL 02234 and by the Cystic Fibrosis Foundation and Thrasher Research Fund.

\begin{abstract}
HBSS, Hanks' balanced salt solution LY, lucifer yellow

LPS, lipopolysaccharide

PMN, polymorphonuclear leukocyte

PMSF, phenylmethylsulfonyl fluoride

TBS, 0.05 M Tris-buffered saline, pH 7.6

FACS, fluorescence-activated cell sorter
\end{abstract}

Optimal host defense requires the interactions of opsonins bound to the surface of the targets of phagocytosis with specific receptors on the surface of the phagocyte. The primary opsonins involved in promoting phagocytosis of bacteria are $\mathrm{IgG}$ and fragments of the third component of complement. These proteins can potentially interact with three different $\mathrm{Fc} \gamma$ receptors $(\mathrm{Fc}$ $\gamma \mathrm{RI}, \mathrm{II}$, and III) and four different C3 fragment receptors (CR1, $-2,-3,-4)$. The specificity and affinity of each of these receptors is unique, and their functional activities differ and may change under different physiologic conditions. Thus, to understand the importance of any individual opsonin, it is critical to know which receptors are expressed on the phagocytic cells the target is likely to encounter in vivo. It has recently become clear that macrophages express $\mathrm{Fc} \gamma$ and complement receptors in a tissuespecific manner $(1-5)$ and that macrophages derived in vitro from peripheral blood monocytes may not express the same receptors as tissue macrophages at sites of infection or inflammation (6). To understand the interaction of the host with an infectious agent, it is therefore necessary to study those opsonins and receptors actually involved in phagocytosis at the site of infection.

In CF, chronic pulmonary infection with Pseudomonas aeruginosa remains a major clinical problem (7). The relationship between the primary CF defect and this infection remains elusive, as do the reasons why the host's defenses fail to eradicate these bacteria. Our previous work has shown that proteases in the lung remove an important complement receptor, $\mathrm{CR} 1$, from phagocytes and an important opsonin, $\mathrm{iC} 3 \mathrm{~b}$, from the bacteria $(8,9)$. This suggests that these mechanisms may severely impair phagocytosis and killing of the bacteria in vivo. Other investigators have shown that IgG antibodies from CF patients have decreased opsonic activity and may actually inhibit or "block" the opsonic activity of other antibodies (10-15). Interestingly, this blocking activity is most apparent with macrophages, and has been reported with neutrophils in only one study (13). To better understand the relative importance of IgG antibody versus comple- 

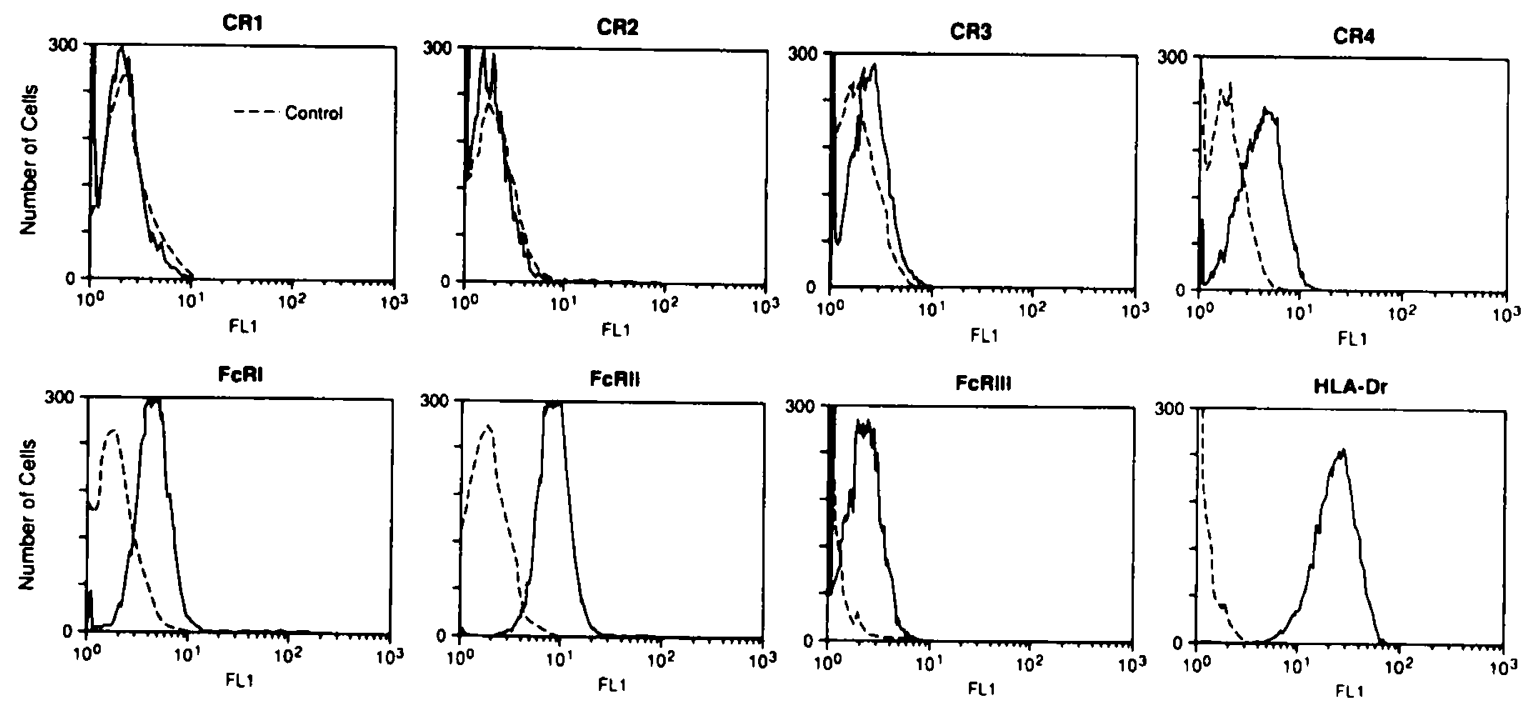

Fig. 1. Receptor expression on normal alveolar macrophages. Separate aliquots of fresh BAL cells were stained with antibody to the indicated receptor or with the appropriate control antibody as described in Materials and Methods. From each aliquot, 10000 cells were analyzed in the FACS, which was gated for cells with volume and $90^{\circ}$ scatter greater than activated neutrophils. For each receptor, the histogram with the antibody to the receptor is shown by the solid line and the control is shown by the dashed line. Results are from a single representative BAL sample.

Table 1. Receptor expression on normal alveolar macrophages and blood neutrophils

\begin{tabular}{lclcc}
\hline Receptor & $\begin{array}{c}\text { CD designation } \\
\text { of antigen }\end{array}$ & Clone & $\begin{array}{c}\text { Fresh BAL macrophages } \\
\text { \% cells positive } \\
\text { (mean } \pm \text { SEM, } n=10)\end{array}$ & $\begin{array}{c}\text { Chemoattractant stimulated } \\
\text { normal blood neutrophils } \\
\text { \% cells positive } \\
\text { (mean } \pm \text { SEM, } n=4)\end{array}$ \\
\hline CR1 & 35 & 3 D9 & $6.8 \pm 1.5$ & $88.4 \pm 3.8$ \\
CR2 & 21 & HB5 & $5.4 \pm 2.1$ & $30.0 \pm 6.6$ \\
CR3 & 11b & M1/70 & $6.7 \pm 1.3$ & $88.8 \pm 4.3$ \\
CR4 & 11 & S-HCL-3 & $34.7 \pm 5.7$ & $63.4 \pm 11.5$ \\
Fc $\gamma$ RI & 64 & 32.2 & $38.5 \pm 7.3$ & $1.3 \pm 1.0$ \\
Fc $\gamma$ RII & 32 & IV.3 & $75.0 \pm 5.1$ & $97.1 \pm 0.2$ \\
Fc $\gamma$ RIII & 16 & NKP 15 & $53.3 \pm 7.6$ & $91.2 \pm 1.2$ \\
HLA-DR & L243 & $95.8 \pm 0.2$ & $0.8 \pm 0.7$ \\
\hline
\end{tabular}

* Receptor expression on fresh normal BAL macrophages $(n=10)$ and FMLP-stimulated purified blood neutrophils $(n=4)$. For each sample, the number of cells with fluorescence greater than the appropriate control for each antibody was determined as described in Materials and Methods.

ment in vivo and to test the hypothesis that macrophages are more sensitive to the effects of "blocking" antibodies because they are more dependent on the interaction of IgG with highaffinity Fc $\gamma \mathrm{R}$ than neutrophils, we determined which Fc $\gamma$ and complement receptors are expressed on alveolar macrophages. We also determined the roles of $\mathrm{IgG}$ and complement in promoting phagocytosis by these macrophages as compared with neutrophils. To be sure that our results reflected the true physiologic situation in vivo, and to avoid alterations in receptor expression due to in vitro handling $(16,17)$, we used fresh BAL cells that were not purified or cultured in vitro. Rather, we used the ability of the flow cytometer to selectively analyze specific types of cells. The results show that fresh human alveolar macrophages express a high-affinity $\mathrm{Fc} \gamma \mathrm{R}$ (type I), but only minimal amounts of the functional complement receptors $C R 1, C R 2$, or CR3. These results correlate with the results of flow cytometry phagocytosis assays on fresh BAL cells, which show that complement deposition on the bacteria fails to enhance phagocytosis by the macrophages, which is mediated primarily by IgG. These observations may provide an explanation for the sensitivity of macrophages to the effects of "blocking antibodies" in CF.

\section{MATERIALS AND METHODS}

Human subjects. Nine stable cystic fibrosis patients with mild to moderate lung disease (mean $\pm \mathrm{SEM} \mathrm{FEV}_{1}=82 \pm 10 \%$ of predicted) were recruited from the Cystic Fibrosis Center of Rainbow Babies and Childrens Hospital. Their mean ( \pm SEM) age was $19.2 \pm 1 \mathrm{y}$. No patient was febrile or taking corticosteroids at the time of study. Ten healthy normal volunteers were recruited from hospital staff and students. Their mean age was $27.7 \pm 2.5$ y. All subjects gave informed consent and all protocols were approved by the Institutional Review Board of University Hospitals of Cleveland. Heparinized peripheral blood samples of 12-24 mL were obtained from each $\mathrm{CF}$ patient just before bronchoscopy was performed. Equivalent blood samples were drawn from normal donors at the same time BAL was performed on normal volunteers.

Bronchoalveolar lavage. Subsegmental lavage was performed using a flexible fiberoptic bronchoscope on awake patients, although some CF patients were lightly sedated with small doses of Demerol and Versed. Local anesthesia was achieved with topical Lidocaine. Multiple aliquots of $30-50 \mathrm{~mL}$ of saline, not exceeding $200 \mathrm{~mL}$ total, were instilled through the bronchoscope. Sixty to $80 \%$ of the instilled fluid was recovered in all cases. Lavage fluid was immediately filtered through surgical gauze to remove mucus plugs and debris. All subsequent steps were performed at $0-4^{\circ} \mathrm{C}$. Lavage cells were harvested by centrifugation at $200 \times g$ for $10 \mathrm{~min}$ and washed three times in HBSS without $\mathrm{Ca}^{2+}$ or $\mathrm{Mg}^{2+}$, supplemented with $0.1 \%$ bovine serum 
Receptor Expression on Normal Blood Neutrophils
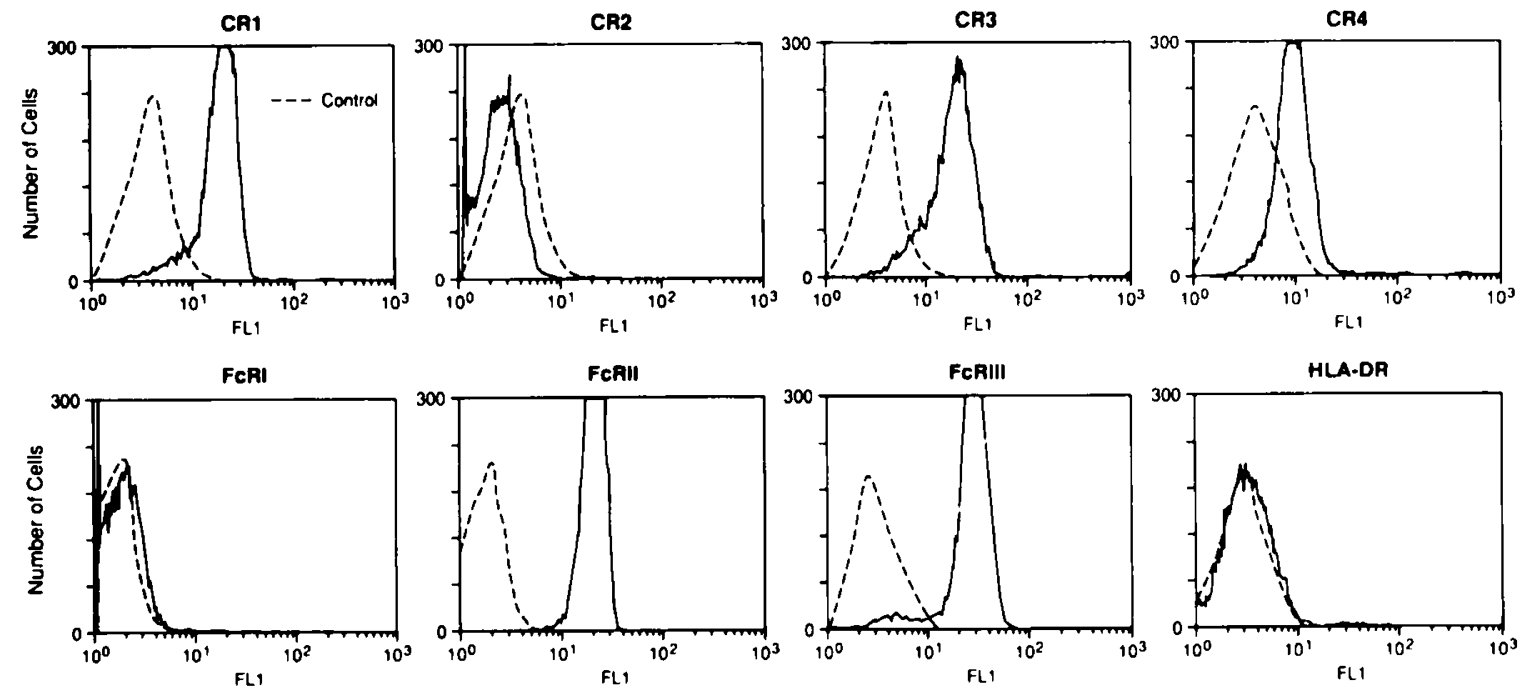

Fig. 2. Receptor expression on purified blood neutrophils stimulated with $10^{-8} \mathrm{M}$ FMLP for $60 \mathrm{~min}$ at $37^{\circ} \mathrm{C}$. Procedure and presentation are the same as in Fig. 1 except that the cytometer gates were set to include the neutrophils.

Table 2. Immunohistologic detection of complement receptors and HLA-DR on normal alveolar macrophages*

\begin{tabular}{lc}
\hline \multicolumn{1}{c}{ Antigen } & $\begin{array}{c}\text { \% Cells positive } \\
\text { (mean } \pm \text { SEM; } n=5)\end{array}$ \\
\hline CR1 & $10.2 \pm 2.9$ \\
CR2 & $4.7 \pm 1.8$ \\
CR3 & $11.8 \pm 1.3$ \\
CR4 & $34 \pm 4.4$ \\
HLA-DR & $85 \pm 3.7$ \\
Control (anticasein) & $5.5 \pm 0.93$ \\
\hline
\end{tabular}

* Receptor expression on morphologically recognizable macrophages in tissue sections of fresh frozen lung specimens. Slides were coded so the observer did not know the antibody with which they were stained. For each antibody, at least 30 cells were graded in each of five lung specimens.

albumin and $10^{-4} \mathrm{M}$ PMSF, then held on ice in this solution. Cell counts were performed using a hemocytometer and differential counts were performed on Wright's stained cytocentrifuge preparations. Viability of lavage cells was assessed by trypan blue exclusion and/or by flow cytometry as described previously (8). Incubation with monoclonal antibodies for determination of receptor expression or with opsonized bacteria for phagocytosis assays was accomplished within $4 \mathrm{~h}$ of performing the BAL.

Isolation of peripheral blood neutrophils. PMN were prepared from heparinized peripheral blood samples as previously described, using Percoll density gradients followed by hypotonic lysis of contaminating erythrocytes (8). Purity and viability of these preparations exceeded 95\%. PMN were resuspended in HBSS without $\mathrm{Ca}^{2+}$ or $\mathrm{Mg}^{2+}$, which was supplemented with $0.1 \%$ gelatin (HBSS gel). Cells were stimulated by incubation with the synthetic chemoattractant FMLP at $10^{-8} \mathrm{M}$ for $1 \mathrm{~h}$ at $37^{\circ} \mathrm{C}$ with intermittent vortexing.

Flow cytometry and immunofluorescent assessment of receptor expression. Separate aliquots of $3 \times 10^{5}$ to $10^{6}$ cells each in HBSS gel were incubated with monoclonal antibodies to Fc $\gamma R, C R$, and HLA-DR, or with control antibodies, on a rotator at $4^{\circ} \mathrm{C}$ for $30 \mathrm{~min}$. The primary antibodies used and the sources from which they were obtained were anti-Fc $\gamma \mathrm{RI}$ (produced by clone 32.2 ) from Medarex Inc., West Lebanon, NH; anti-Fc $\gamma$ RII (produced by clone IV.3) from Dr. Michael Fanger, Dartmouth University, Hanover, NH; anti-Fc $\gamma$ RIII (Anti-leu 11a) from Becton Dickinson, Mountain View, CA; anti-CRI (produced by clone 3D9)
(8, 18); anti-CR2 (produced by clone HB-5) from Becton-Dickinson; anti-CR3 (Mac 1 antigen on $\mathrm{CD} 1 \mathrm{lb}$, produced by clone M1/70) (8, 17); anti-Leu M5 (CD11c) from Becton Dickinson; and anti-HLA-DR from Becton Dickinson. The antibodies to Fc $\gamma$ RIII and HLA-DR were purchased as FITC conjugates. When these antibodies were used, FITC-conjugated nonimmune antibodies of the same isotypes (IgG1 and IgG2a), which were also purchased from Becton Dickinson, were used as controls. For nonconjugated primary antibodies, an irrelevant primary antibody, mouse IgGl anticasein, was used as a control. This antibody was the kind gift of Dr. Charlotte Kaetzel, Case Western Reserve University. No differences were observed when control nonconjugated antibodies of other isotypes were substituted for the anticasein monoclonal. After reaction with primary antibody, cells were washed three times with HBSS containing $0.1 \%$ bovine serum albumin, $10^{-4} \mathrm{M}$ PMSF, and $0.01 \% \mathrm{NaN}_{3}$ and then stained with FITC-conjugated $F\left(a^{\prime}\right)_{2}$ fragments of goat antimouse immunoglobulin as previously described $(8,18)$. Cells were then washed three additional times and held on ice in PBS containing $0.01 \% \mathrm{NaN}_{3}$ until examination in the flow cytometer, generally within $1 \mathrm{~h}$.

Flow cytometry was performed with a Becton Dickinson FACS analyzer equipped for measurement of green and red fluorescence, light scatter at $90^{\circ}$, and true cell volume by the electrolyte displacement method. All data were recorded and processed by a dedicated Consort 30 computer. Analysis gates were set using true cell volume and $90^{\circ}$ scattered light characteristics of resting and FMLP-stimulated peripheral blood neutrophils from each individual. BAL cells falling within these gates were considered neutrophils, cells with greater scatter and volume were considered macrophages. Each set of gates was then used to selectively analyze 5000 to 10000 cells of each type from the BAL samples of the CF patients. Neutrophils and macrophages were analyzed concurrently in all CF patients. Only macrophages could be analyzed in BAL samples of the normal donors because there were not sufficient neutrophils in these samples (8). Background fluorescence was determined on samples reacted with the anticasein control antibody and the same FITC conjugate, or with the isotype matched directly conjugated control antibodies for anti-Fc $\gamma$ RIII and anti-HLA-DR. The percentage of positive cells in the sample stained with any given primary antibody was then determined by computerized subtraction of the background histogram, or by determining the number of cells with fluorescence greater than (to the right of) a discriminator set to include (to its 

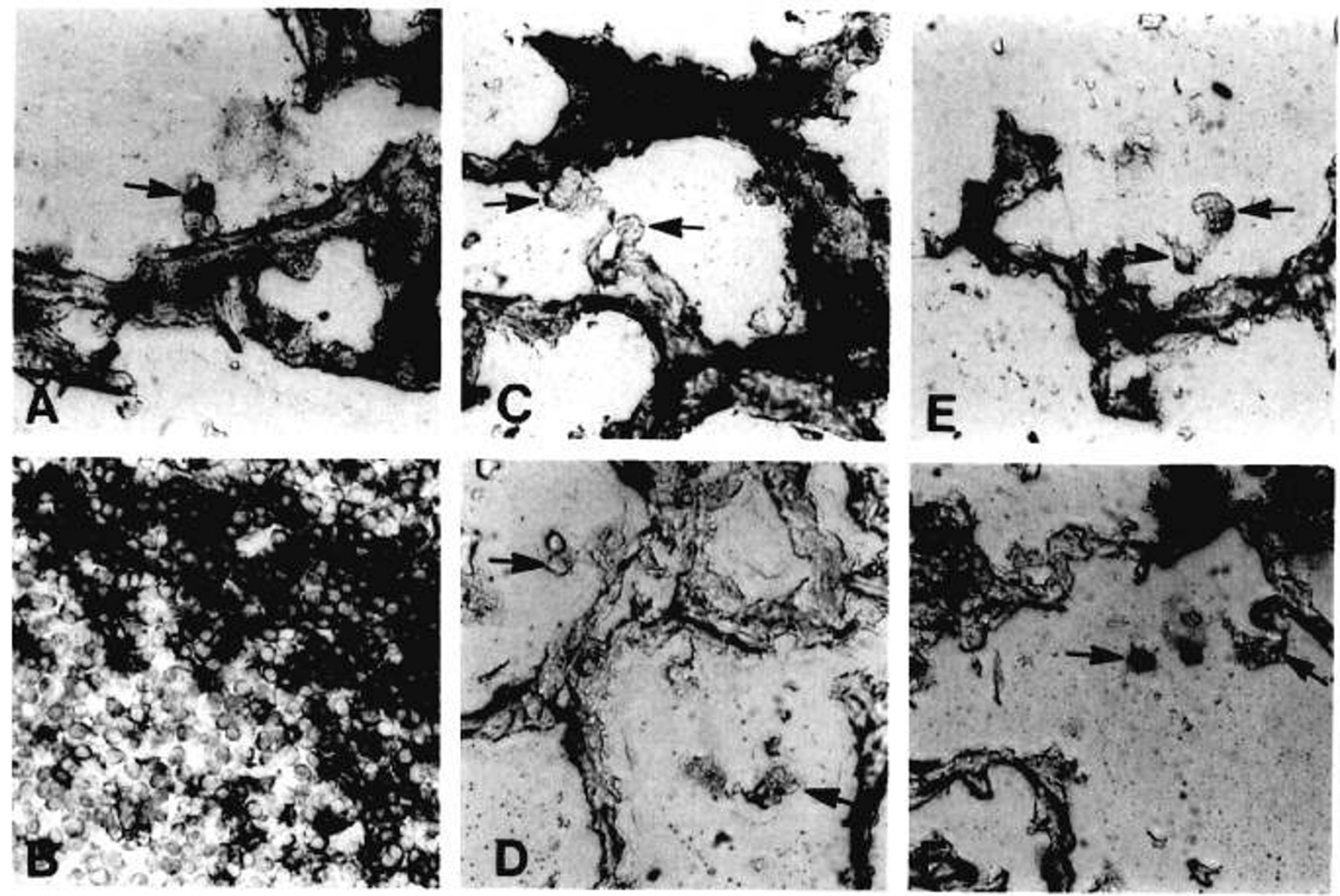

Fig. 3. Phase contrast photomicrographs of sections stained by immunoperoxidase reveal intense staining of alveolar macrophages (arrows) with anti-HLA-DR $(A)$, but not with anti-CR1 $(C)$, anti-CR2 $(D)$, or anti-CR3 $(E)$. Weak staining was seen in $30 \%$ of alveolar macrophages with antiCR4 $(F)$. Positive controls included a section of lymph node stained with anti-CR1 $(B)$. Follicular lymphocytes (upper right) but not paracortical lymphocytes (lower left) reveal intense staining with anti-CR1 $(B)$ and CR2 (not shown). Original magnification $\times 150$.

left) $98 \%$ of the cells stained with the control antibody. In all cases, results determined with these two different methods agreed within $5 \% . t$ tests were used to evaluate the statistical significance of differences in receptor expression between $\mathrm{CF}$ and normal cells.

Immunohistochemical staining of fixed lung tissue. Cryostat sections $(4 \mu \mathrm{m})$ of normal lungs obtained at autopsy within $12 \mathrm{~h}$ postmortem were air dried, fixed by immersion in acetone for $10 \mathrm{~min}$, and equilibrated and washed three times in TBS. Sections of spleen, lymph node, and kidney were also employed as specificity controls. Fifty microliters of each monoclonal antibody were then applied to the sections. Primary murine antibodies were diluted at 1:5 for immunofluorescence and 1:20 for immunoperoxidase from the commercial stock solutions, with the exception of anti-CR 1 (3D9), from ascites, and anti-CR3 (Mac1) hybridoma culture supernatant. These latter antibodies were used at 1:50 and undiluted, respectively, for immunofluorescence and at 1:200 and 1:4, respectively, for immunoperoxidase. All dilutions were in TBS; incubations with primary antisera were performed at $25^{\circ} \mathrm{C}$ for $30 \mathrm{~min}$ for immunofluorescence and at $4^{\circ} \mathrm{C}$ for $22 \mathrm{~h}$ for immunoperoxidase. Slides for immunofluorescence were subsequently stained, after three washes with TBS, with a 1:10 dilution of an Fc-specific fluoresceinated goat IgG fraction of anti-mouse IgG, applied for $30 \mathrm{~min}$ at $25^{\circ} \mathrm{C}$. For immunoperoxidase, slides were stained with the Vectastain Elite Mouse Kit (Vector Laboratories, Burlingame, CA), using biotinavidin-peroxidase methods exactly as recommended by the manufacturer. All slides were mounted in $50 \%$ glycerol in PBS, pH 7.5. Coded specimens from five lungs were examined microscopically by a single experienced observer who was unaware of the antibodies used. Both immunoperoxidase and immunofluores- cent stained specimens were examined in parallel, and excellent agreement was obtained with the two different procedures.

Bacteria, opsonization, and phagocytosis assays. Nonmucoid Fisher-Devlin immunotype (FT) 1 Pseudomonas aeruginosa and a mucoid strain were clinical isolates from CF patients obtained from the CF Core Center at Case Western Reserve University. Bacteria from overnight broth cultures were heat killed at $60^{\circ} \mathrm{C}$ for $30 \mathrm{~min}$, washed twice with PBS containing $\mathrm{Ca}^{2+}, \mathrm{Mg}^{2+}$, and $0.2 \%$ glucose by centrifugation at $12000 \times g$ for $10 \mathrm{~min}$, and labeled with the fluorescent reagent LY (Sigma) by incubation for $90 \mathrm{~min}$ at room temperature as described by Sveum et al. (19). Bacteria were then washed three times and aliquots were stored frozen in suspension at $-80^{\circ} \mathrm{C}$ as previously described (9). Bacteria were thawed just before use, washed in PBS-glucose, then incubated on a rotator at $10^{7} / \mathrm{mL}$ for $45 \mathrm{~min}$ at $37^{\circ} \mathrm{C}$ with the indicated concentration of a previously described $(20)$ murine IgG3 monoclonal antibody specific for the $O$-side chain of FT 1 LPS. Alternate sources of antibody included a polyvalent human intravenous immunoglobulin prepared from plasma of normal donors with high titers of anti-Pseudomonas antibodies (Pseudomonas Immune Globulin, Cutter, Inc., Berkeley, CA) and polyvalent human hyperimmune globulin prepared from the plasma of volunteers immunized with a Pseudomonas polysaccharide conjugate vaccine (Nosocuman, Dr. Alan Cross, Walter Reed Army Institute of Research, Washington, DC). These preparations had equivalent titers of antibody to Pseudomonas LPS as determined in an enzyme-linked immunosorbent assay (20). In some experiments a murine $\mathrm{IgG} 2 \mathrm{~b}$ monoclonal against Pseudomonas mucoid exopolysaccharide, kindly supplied by Dr. Gerald Pier, Channing Laboratories, Boston, MA (21), was employed with a mucoid strain of bacteria. In indicated 


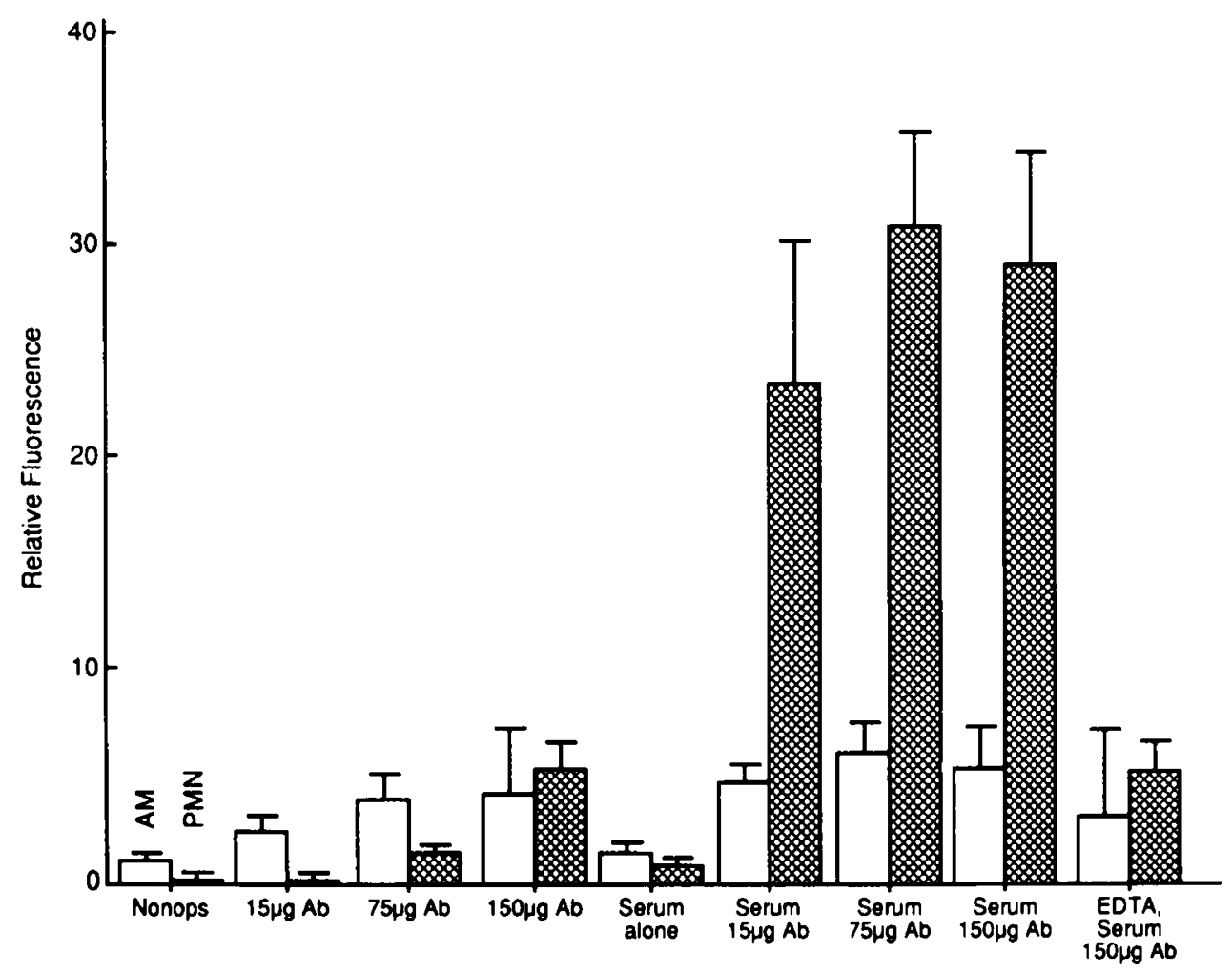

Fig. 4. Phagocytosis of fluorescent tagged $P$. aeruginosa by fresh normal alveolar macrophages and FMLP-stimulated purified blood neutrophils. Washed, LY-tagged FT 1 bacteria were preopsonized as described in the figure when washed again and incubated with BAL cells (open bars) or neutrophils (cross-hatched bars) as described in Materials and Methods. The antibody used was a monoclonal murine IgG3 against the LPS $O$-side chain high molecular weight polysaccharide. Note that $15 \mu \mathrm{g} / \mathrm{mL}$ corresponds to $10^{-7} \mathrm{M}$. Mixtures were analyzed in the FACS with gates set for macrophages or stimulated neutrophils respectively. From each sample, 5000 cells were analyzed and the arithmetic mean fluorescence was determined by the Consort 30 computer. The mean fluorescence for BAL cells and neutrophils run in the absence of bacteria was subtracted as background from other samples with the same type of phagocyte. Results shown are the mean \pm SEM of six experiments.

experiments, bacteria were concurrently treated with antibody and with pooled normal human serum as a source of complement ( $5 \%$ final serum concentration). This serum pool contained no detectable antibody to $P$. aeruginosa as determined with a 1:100 dilution in an enzyme-linked immunosorbent assay using whole mucoid bacteria as an absorbent. Sera from chronically infected CF patients are generally positive in this assay at dilutions $\geq 1: 10000$. Opsonization with serum was also performed on a rotator for $45 \mathrm{~min}$ at $37^{\circ} \mathrm{C}$. Bacteria were then washed twice and resuspended in $\mathrm{HBSS}$ containing $\mathrm{Ca}^{2+}, \mathrm{Mg}^{2+}$, and $0.1 \%$ gelatin. Although we did not quantify the amounts of $\mathrm{C} 3 \mathrm{~b}$, iC $3 \mathrm{~b}$ and $\mathrm{C} 3 \mathrm{~d}$ on the bacteria used in these studies, our previous results (9) suggest that all three of these fragments are fixed onto $P$. aeruginosa under these conditions. Phagocytosis assays were performed as described previously (9). Briefly, $2 \times 10^{7}$ opsonized, LY-tagged bacteria were incubated at $37^{\circ} \mathrm{C}$ with $10^{\circ} \mathrm{BAL}$ cells or isolated peripheral blood PMN in a total volume of $0.24 \mathrm{~mL}$ of HBSS containing $\mathrm{Ca}^{2+}, \mathrm{Mg}^{2+}$, and $0.1 \%$ gelatin. Parallel mixtures were incubated at $0^{\circ} \mathrm{C}$ to allow estimation of the proportion of bacteria attached to the surface of the phagocytes but not ingested. After $30 \mathrm{~min}$ of gentle agitation, $2.0 \mathrm{~mL}$ of cold PBS was added and phagocytic cells were separated from most of the free bacteria by three differential centrifugations at $180 \times$ $g$ for $5 \mathrm{~min}$. The cell mixtures were analyzed by fluorescence flow cytometry using volume and $90^{\circ}$ light scatter gating as described above, which also served to eliminate any remaining free bacteria from analysis. From each sample, the fluorescence of 5000 cells within each set of volume and light scatter gates was recorded by the cytometer, and the arithmetic mean fluorescence was determined automatically by the computer, allowing linear comparison of fluorescence data.

\section{RESULTS}

Opsonin receptors on fresh alveolar macrophages from normal subjects. A representative set of fluorescence histograms for separately immunostained aliquots of fresh bronchoalveolar lavage macrophages from a normal donor is shown in Figure 1. The result for each antireceptor antibody is shown by the solid line, and the result for the corresponding control is shown by the dashed line in each block. The fluorescence value on the $x$ axis is directly proportional to the number of antireceptor monoclonal antibody molecules bound to each cell, and hence also to the number of receptor molecules expressed on each cell (22). The values on the $y$ axis show the number of cells with each fluorescence value. It is readily apparent that the histograms for anti-CR 1 and anti-CR2 do not differ appreciably from the corresponding controls, although there is some positive fluorescence for anti-CR3 and a larger difference in the positive fluorescence for anti-CR4 as compared with its control. In contrast, there is relatively less overlap between the histogram for anti-Fc $\gamma \mathrm{RI}$ with its control, even less for anti-Fc $\gamma$ RII and III, and essentially no overlap with anti-HLA-DR. As summarized in Table 1, the results from this subject taken together with results from 9 other normal subjects show that fewer than $10 \%$ of alveolar macrophages exhibited specific binding of anti-CR1, anti-CR2, or antiCR 3 antibodies. This suggests that expression of these receptors (CR1, CR2, and CR3) is negligible. In contrast, about $30-40 \%$ of cells express CR4 and Fc $\gamma \mathrm{RI}$, with mean fluorescence values of less than 10, whereas the percentages of cells expressing Fc $\gamma$ RII and III are higher. It should be noted that these figures may underestimate the actual percentage of positive cells, particularly for the Fc $\gamma R$ because for Fc $\gamma R I$, for example, the macrophages stain as a single population, but there is some overlap with the 

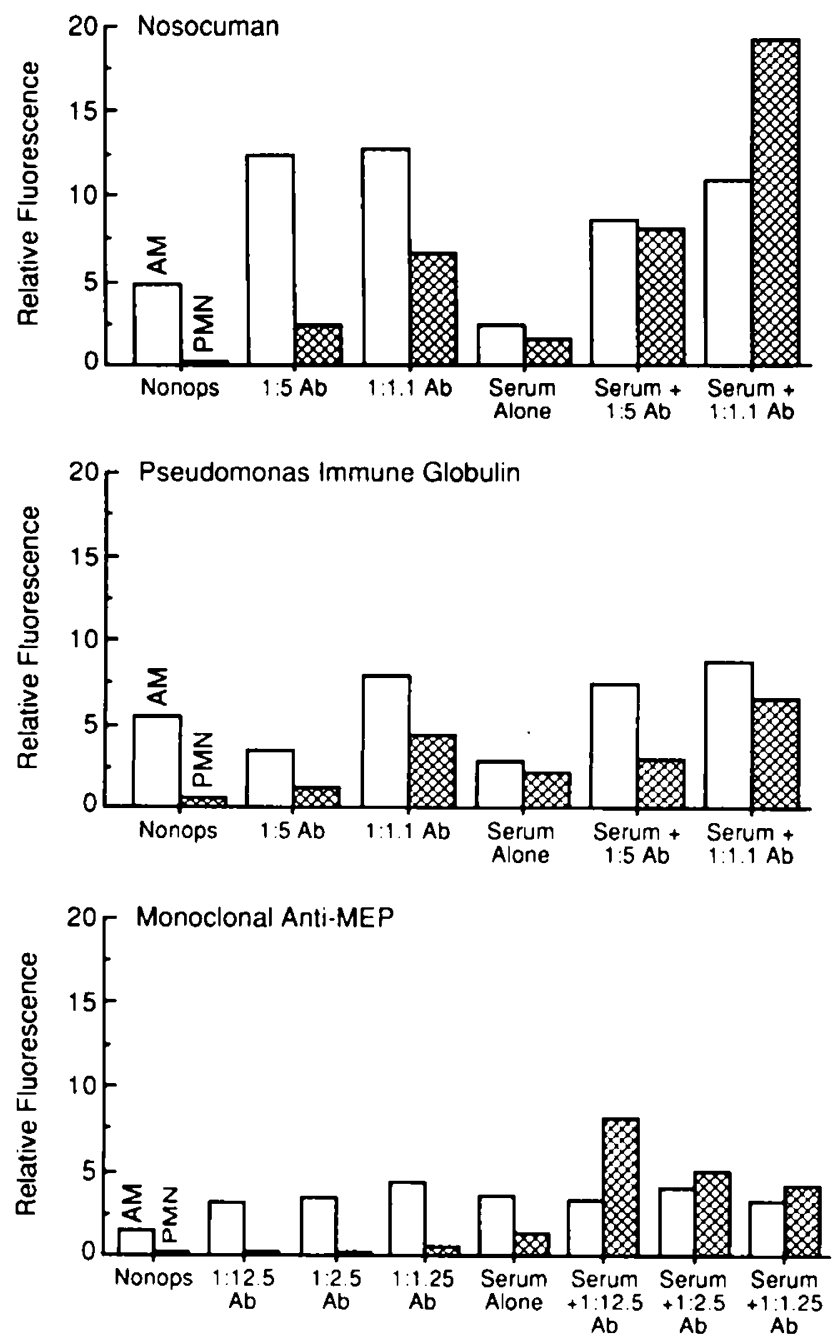

Fig. 5. Effects of different sources of antibody on phagocytosis of fluorescent tagged $P$. aeruginosa by fresh normal alveolar macrophages (open bars) and purified blood neutrophils (cross-hatched bars). Procedures are the same as in Figure 3 except that the indicated sources of antibody were substituted for the monoclonal antibody used in that study. The upper two panels show the effects of human polyclonal antisera; the lower panel is for a monoclonal murine $\mathrm{IgG} 2 \mathrm{~b}$ antimucoid exopolysaccharide. With this antibody only, a mucoid stain of $P$. aeruginosa was used. Results shown are for a single representative experiment.

histogram for the control antibody, suggesting that some cells expressing relatively few receptors may have fluorescent values that overlap with cells that have the highest nonspecific binding of the control antibody. Similar observations have been reported by Kindt $e t$ al. (5). There were no subpopulations of macrophages as defined by light scatter and/or volume characteristics that had distinctive differences in $\mathrm{CR}$ or Fc $\gamma \mathrm{R}$ expression in either the CF or normal BAL specimens. The expression of HLA-DR is essentially universal. The latter observation confirms that the cells being analyzed are macrophages because neutrophils and epithelial cells do not express class II histocompatibility antigens (23). Comparable fluorescence histograms for chemoattractantstimulated normal blood neutrophils are shown in Figure 2. A summary of the results with chemoattractant stimulated neutrophils for this subject and 3 other normal donors is also provided in Table 1 as a comparison. These results are consistent with our results for CR expression on stimulated blood neutrophils published previously (8). The chemoattractant exposure mimics that to which PMN would be exposed as they are recruited from the circulation to the site of infection $(8,18)$. Differences from the results with the macrophages are readily apparent: most notably, the neutrophils are markedly positive for CR1 and CR3, only slightly positive for Fc $\gamma \mathrm{RI}$, and very markedly positive for Fc $\gamma$ RII and Fc $\gamma$ RIII. In further contrast to the macrophages, the neutrophils are uniformly negative for HLA-DR.

Immunohistochemistry of CR expression on fixed lung tissue. To be sure that our findings on BAL cells were representative of alveolar macrophages in situ, and to confirm the relative lack of expression of CR1, 2 or 3 in vivo, we performed immunohistochemical analysis of fixed lung tissue using the same antibodies. The results of sections from 5 lungs, coded so that the observer did not know which antibody was used, are summarized in Table 2 , and a representative set of micrographs is shown in Figure 3. Most morphologically recognized intralveolar macrophages (arrows) stained intensely for HLA-DR. Alveolar macrophages were uniformly negative for CR2 and only weakly and infrequently positive for CR1 and CR3, in good agreement with our FACS data. The percentage of cells positive for CR4 was also in good agreement with the FACS data. In contrast, using lymphoid tissue for positive controls showed strong staining of follicular cells in lymph nodes and spleen for CR1 and CR2, and of medullary macrophages in lymph nodes for CR1, CR3, and CR4. The positive staining for CR1 and CR2 in these control tissues suggests that their absence from the alveolar macrophages is not due to postmortem proteolysis.

Role of $\mathrm{IgG}$ and $C 3$ fragments in promoting phagocytosis of $\mathrm{P}$. aeruginosa by fresh alveolar macrophages from normal subjects. Having determined the pattern of opsonin receptor expression on the BAL macrophages, we next evaluated the relative efficacy of IgG and complement in promoting phagocytosis of $P$. aeruginosa by these cells as compared with chemoattractant-stimulated blood PMN. We first used a mouse monoclonal IgG3 antibody to the high molecular weight polysaccharide O-side chain of the LPS of Fisher-Devlin immunotype $1 P$. aeruginosa with the homologous bacteria as the targets. These assays were performed with bacteria rendered fluorescent with lucifer yellow, which does not lose its fluorescence in intracellular acidified compartments. Thus, greater relative fluorescence indicates a greater number of bacteria associated with the phagocyte. Separate experiments performed at $0^{\circ} \mathrm{C}$ to allow attachment but not internalization of opsonized bacteria showed that in every case, internalized bacteria accounted for $>90 \%$ of the total cell-associated bacteria. Free bacteria were excluded from the analysis by the volume and light scatter gates which were set for each type of phagocyte. For comparison, chemoattractant-stimulated blood neutrophils were included in parallel in all experiments. Control experiments showed that incubation of normal and $\mathrm{CF}$ cells under the conditions of phagocytosis assay did not alter their patterns of receptor expression.

There was little phagocytosis of nonopsonized bacteria by either alveolar macrophages (open bars) or neutrophils (PMN, cross-hatched bars), as shown at the left of Figure 4 . With the addition of the lowest concentration of specific antibody, $15 \mu \mathrm{g} /$ $\mathrm{mL}\left(10^{-7} \mathrm{M}\right)$, phagocytosis by the alveolar macrophages increased from $0.57 \pm 0.04$ to $2.5 \pm 0.35$ ( $p<0.001$ by $t$ test), although there was no increment in phagocytosis by the neutrophils. With increasing inputs of antibody, phagocytosis by both types of cells increased, but the macrophages showed little additional enhancement of phagocytosis when the antibody concentration was increased from $75 \mu \mathrm{g} / \mathrm{mL}$ to $150 \mu \mathrm{g} / \mathrm{mL}$, consistent with saturation of high affinity Fc $\gamma \mathrm{R}$. In contrast, the PMN did not appear to exhibit a saturation phenomenon at these concentrations, and the highest input of antibody gave the greatest enhancement of phagocytosis.

To determine the effects of complement, we incubated the bacteria, with or without antibody, with $5 \%$ pooled normal human serum. These results are shown in the right half of Figure 4. With either macrophages or PMN, serum alone did not cause much enhancement of phagocytosis above the minimal level seen with nonopsonized bacteria, confirming that this serum pool did not contain functional antibody to these bacteria. When 

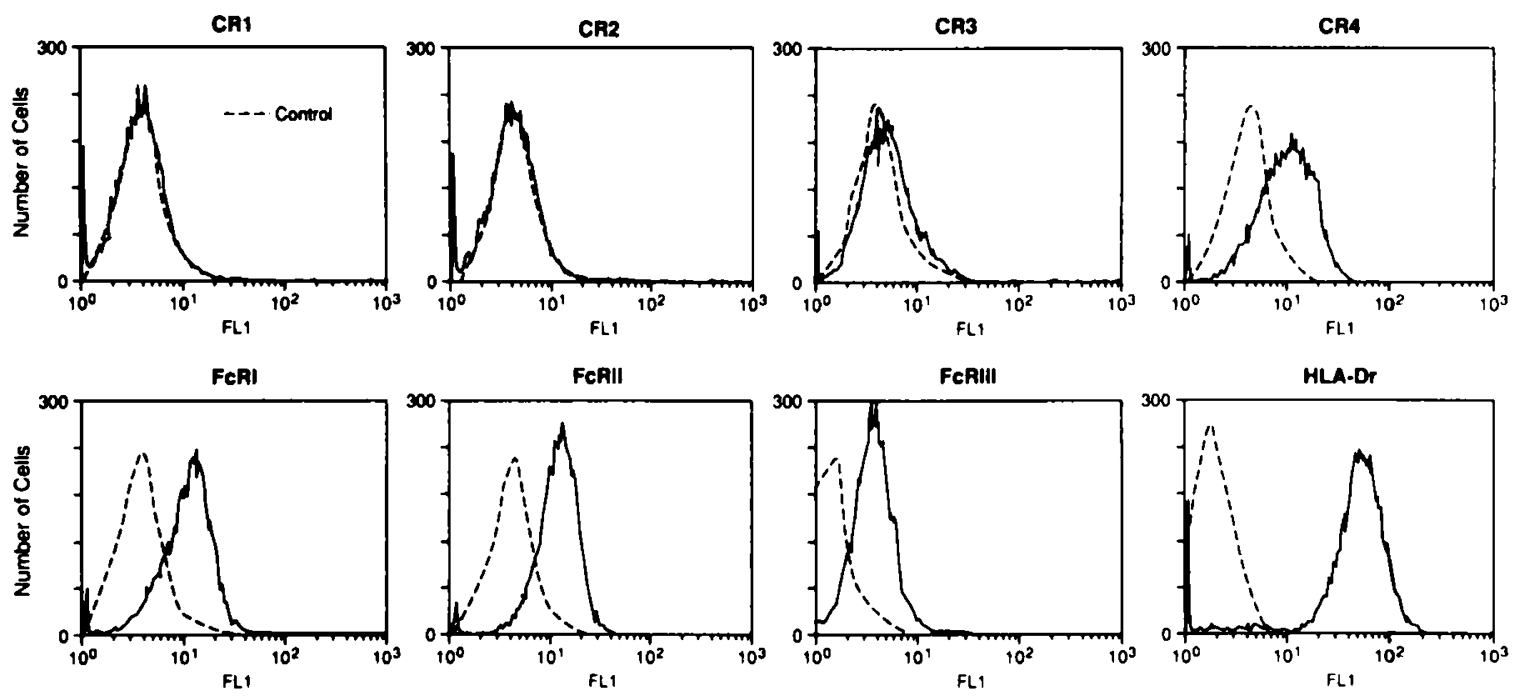

Fig. 6. Receptor expression on fresh BAL macrophages from a CF patient. Procedures and presentation are the same as in Figure 1.

Table 3. Receptor expression on CF BAL macrophages*

\begin{tabular}{lc}
\hline Receptor & $\begin{array}{c}\text { \% Cells positive } \\
\text { (mean } \pm \text { SEM, } n=7 \text { ) }\end{array}$ \\
\hline CR1 & $10.5 \pm 0.6$ \\
CR2 & $0 \pm 1.3$ \\
CR3 & $4.9 \pm 2.6$ \\
CR4 & $20.9 \pm 7.0$ \\
Fc $\gamma$ RI & $20.0 \pm 7.0$ \\
Fc $\gamma$ RII & $32.2 \pm 7.3$ \\
Fc $\gamma$ RIII & $30.0 \pm 7.0$ \\
HLA-DR & $80.3 \pm 2.9$ \\
\hline
\end{tabular}

* Receptor expression on fresh BAL macrophages from CF patients $(n=7)$. Procedures and presentations are as in Table 1 .

serum was used together with $15 \mu \mathrm{g} / \mathrm{mL}$ antibody, phagocytosis by the macrophages doubled, reaching 4.4 relative fluorescence units. In contrast, the addition of serum to this small amount of antibody had a much more dramatic effect with the neutrophils, increasing phagocytosis over 20 -fold, from less than 1 to over 23 units. In the presence of serum, higher inputs of antibody caused even greater enhancement of phagocytosis by the neutrophils, but there was little additional effect with the macrophages. To be sure that the effect of serum was, in fact, due to complement fixation, we performed control experiments in which opsonization was performed in the presence of $10 \mathrm{mM}$ EDTA. As seen on the extreme right, this abolished the enhancement of neutrophil phagocytic activity caused by serum, and the results were not different from those obtained with the same input of antibody alone $(150 \mu \mathrm{g})$. Similar results were also obtained when the serum was heat-inactivated. The fact that EDTA and heat inactivation abolished the effects of serum in the opsonization step, before the opsonized bacteria were washed and added to the phagocytes, provides strong evidence that these effects are due to complement.

To rule out the possibility that the observed results were peculiar to the isotype of mouse antibody used or the specific epitope it recognizes on the bacteria, we performed similar experiments with two sources of polyclonal human immunoglobulin. We also performed similar experiments with a mucoid strain of $P$. aeruginosa and a mouse monoclonal antibody specific for mucoid exopolysaccharide. As shown in Figure 5, the results for both alveolar macrophages and PMN are qualitatively quite similar to those reported above regardless of whether the bacteria were opsonized with Nosocuman, a human polyclonal hyperimmune globulin prepared from plasma of normal volunteers immunized with pseudomonas polysaccharides conjugated to a protein carrier (top panel), or with the mouse IgG2b monoclonal to mucoid exopolysaccharide, even though with the latter antibody a mucoid strain of bacteria was used (bottom panel). The results with the immune globulin prepared from plasma of donors with naturally occurring high titers of anti-Pseudomonas antibodies (middle panel) differ slightly, in that there is less overall enhancement of phagocytosis and less dramatic enhancement of PMN phagocytosis in the presence of serum as well. Even with this preparation, however, increasing the antibody concentration increased phagocytosis by the macrophages more than with the PMN, and the addition of serum did not increase phagocytosis by the macrophages more than antibody alone. The quantitative differences between the results with these antibodies and those in Figure 4 may relate to the subclasses and/or affinities of the specific antibodies in these preparations. More importantly, the qualitative similarity of the results with these other sources of antibody and with a different strain of bacteria to the results obtained with the mouse monoclonal antibody to the pseudomonas LPS $O$-side chains used above suggests that our observations are primarily related to the pattern of opsonin receptor expression on the phagocytes, rather than to peculiarities of any given source of antibodies or bacterial strain.

Receptor expression and phagocytosis by $C F$ alveolar macrophages. Because one of our major goals is to better understand the actual opsonin-receptor interactions in the milieu of the chronically infected lung in cystic fibrosis, we next studied bronchoalveolar lavage samples from patients with that disease. Although as the chronic Pseudomonas infection progresses, neutrophils become the predominant type of cell recoverable on bronchoalveolar lavage, we were able to obtain BAL specimens with a mean $( \pm$ SEM) content of $36.6 \pm 4.5 \%$ macrophages and 60 $\pm 4.9 \%$ neutrophils by studying patients with stable mild disease. The ability of the flow cytometer to selectively analyze each of these types of cells was then used to determine how they could perform in vivo, avoiding in vitro separation or culturing techniques that might alter their patterns of receptor expression or phagocytic function.

CF BAL macrophages fell within the same volume and $90^{\circ}$ light scatter ranges as those from normal subjects. The fluorescence histograms for receptor expression on CF BAL macrophages are quite similar to those obtained with normal macrophages, as shown in the representative histograms in Figure 6. The results for seven patients are summarized in Table 3 . There 


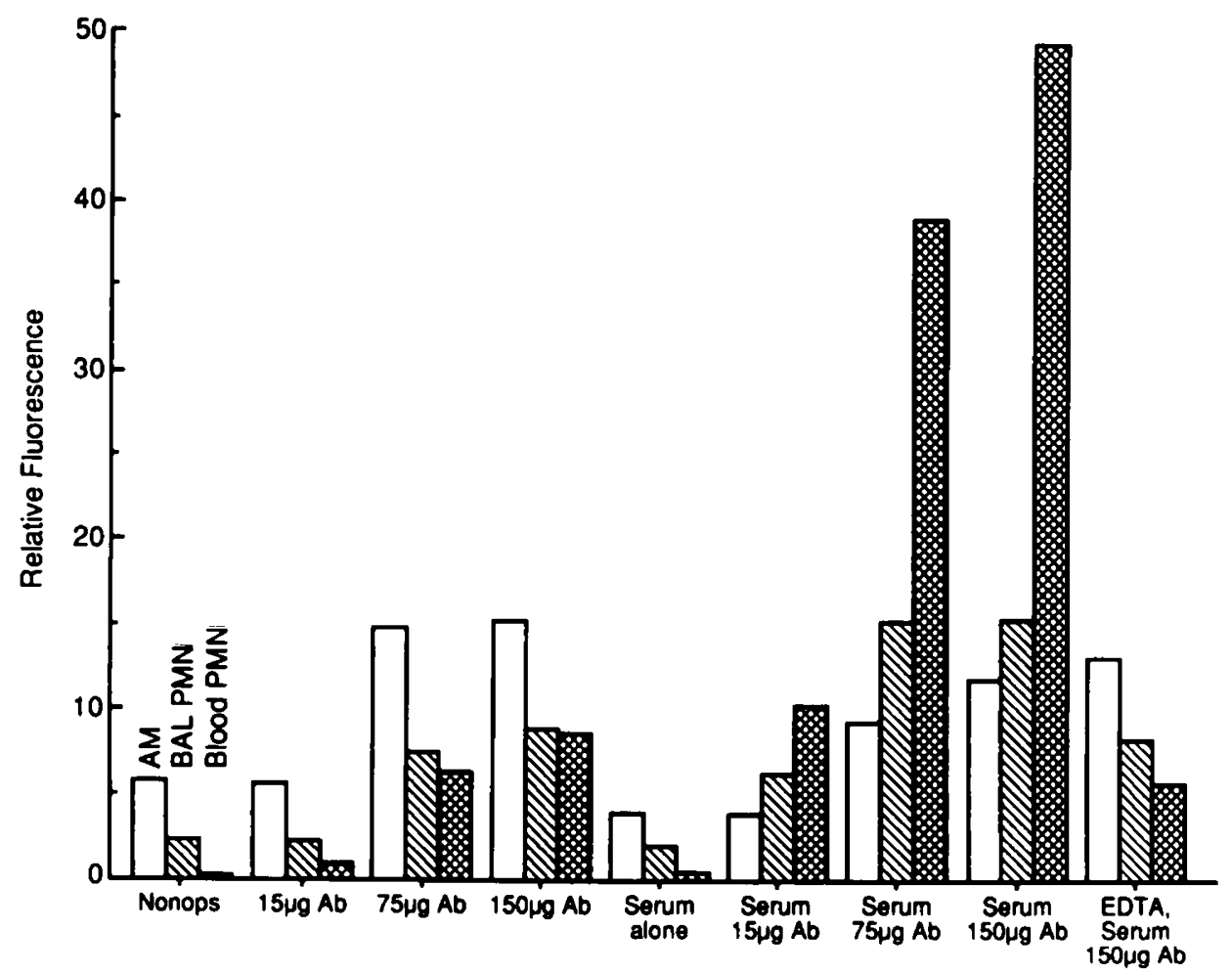

Fig. 7. Comparison of phagocytosis of fluorescent-tagged FT 1 P. aeruginosa by fresh BAL macrophages (open bars), fresh BAL neutrophils (hatched bars) and FMLP-stimulated blood neutrophils (cross-hatched bars) from a single CF patient. Procedures are as in Figure 4, except that each BAL sample was analyzed twice, once with gating on neutrophils and once with gating on macrophages. Results are for a single representative experiment.

were no statistically significant differences in expression of the complement receptors or Fc $\gamma \mathrm{RI}$, but the percentage of CF cells expressing Fc $\gamma$ RII, Fc $\gamma$ RIII, and HLA-DR were lower than the percentage of normal BAL macrophages expressing these proteins $(p<0.001,<0.05$, and $<0.001$ respectively). The decreased expression of Fc $\gamma R$, especially Fc $\gamma$ RII, on the macrophages of some of the CF patients may reflect internalization during phagocytosis in vivo, and/or the effects of proteases $(8,25)$. We have not yet directly evaluated the extent to which either or both of these mechanisms (or others as well) contribute to the observed decrease in Fc $\gamma \mathrm{R}$ expression. Receptor expression on BAL neutrophils was analyzed concurrently with that on the macrophages in each CF BAL specimen. The results are essentially identical to those we obtained previously and have already presented in detail in ref. 8. Basically, the CF BAL neutrophils are quite similar to chemoattractant-stimulated blood neutrophils (Fig. 2), except for greatly reduced CRI and Fc $\gamma$ RIII expression. In no case did CF blood or BAL neutrophils stain positively for Fc $\gamma \mathrm{RI}$.

The effects of antibody and complement in enhancing phagocytosis by CF BAL macrophages are shown in the open bars in Figure 7 , and are also similar to those obtained with normal macrophages. Once again, phagocytosis appeared to be optimized at the intermediate input of antibody, with additional antibody causing only a slight additional increment. Serum alone had no effect, and the addition of serum to the antibody caused essentially no enhancement above the level seen with each input of antibody alone. Results with eight other CF patients' alveolar macrophages were also similar (not shown). Results with chemoattractant-stimulated CF blood neutrophils, shown in the crosshatched bars, were similar to those for chemoattractant-stimulated blood neutrophils from normal donors shown in Figure 4. The results with CF BAL neutrophils, shown in the middle bar in each group, differ from those with the blood neutrophils from the normal donors (shown in Fig. 4) or from those obtained with blood neutrophils from the same CF patient. The most striking difference is that the CF BAL neutrophils, in contrast to the blood neutrophils, show little enhancement of phagocytosis upon the addition of serum. This is consistent with the markedly decreased CR1 expression on CF BAL neutrophils we observed in these and previous studies (8) and emphasizes the pathophysiologic significance of those earlier observations.

\section{DISCUSSION}

The problem of chronic lung infection with $P$. aeruginosa in CF remains enigmatic. Because CF patients do not have an increased frequency of infection outside of the respiratory tract, and no systemic immunologic defect has been identified, we have focused on studying the local host defense mechanisms as they function in situ, in the milieu of the chronically infected lung. The major cells involved in defense of the lung against bacterial infection are neutrophils and alveolar macrophages. The phagocytic activity of these cells is primarily determined by the receptors they express for opsonins on the surface of their targets, and by the functional capabilities of these receptors. Both the expression and function of these receptors may be altered dramatically by a wide variety of extracellular signals. For example, complement receptor expression by neutrophils is rapidly and markedly increased by chemoattractants (18). Macrophages in tissues also express a very different array of complement and immunoglobulin receptors than do the blood monocytes from which they are derived $(3,4)$. Monocytes, when cultivated in vitro, may express different receptors depending on which supplements are added to the culture media $(6,16)$. Moreover, 5fold changes in Fc $\gamma$ receptor expression and differences in phagocytic activity can be induced during isolation of monocytes (17). Although the factors that determine receptor expression in vivo have not been completely defined, it is clear that receptor expression varies in a tissue-specific manner $(3,4)$. In the present work, we have taken advantage of the ability of the flow cytometer to analyze selectively individual types of cells in complex 
mixtures, and have used immunofluorescent staining to determine $\mathrm{Fc} \gamma$ and complement receptor expression, and fluorescent tagged bacteria to determine the phagocytic activity of these cells, all within a few hours of their removal from the lung. This approach obviates the need for adherence, other purification procedures, or artificial culture media that may alter receptor expression and/or function in vitro $(16,17)$. Thus we believe that our results are indicative of the true state of these cells as they function in vivo at the site of infection.

With normal alveolar macrophages, we found minimal or no expression of complement receptors CR1, CR2 or CR3. This differs markedly from blood monocytes either at rest or after stimulation or culture in vitro (2), but is similar to reports from other laboratories for alveolar macrophages $(3,4)$. Studies using the same monoclonal antibodies to stain fresh-frozen lung tissue specimens also failed to show any appreciable staining, confirming that the apparent lack of expression of these receptors on cells obtained by bronchoalveolar lavage was, in fact, representative of their status in vivo. We did find moderate staining with the Leu-M5 antibody, which recognizes the CD1lc-chain of p150/95, the "third" member of the Mac-1/LFA-1 family of leukocyte surface adherence proteins (24), which has been identified tentatively as CR4 (4). This is also consistent with the results of Myones et al. (4), who found that p150/95 was the most abundant member of this family on tissue macrophages. This is in marked contrast to neutrophils, which, after exposure to chemoattractants as would occur during their migration into tissues in vivo, express relatively large amounts of $C R 1$ and CR3. We also found differences in the expression of $F c \gamma R$ on alveolar macrophages versus neutrophils, since the macrophages expressed the high affinity IgG receptor, Fc $\gamma \mathrm{RI}$, in addition to Fc $\gamma$ II and III; whereas the neutrophils express only Fc $\gamma$ RII and III $(5,8,18,25)$. Although it has been reported that blood neutrophils from some subjects with acute streptococcal infection express Fc $\gamma \mathrm{RI}$ (26), this receptor was not found on blood or BAL neutrophils from the stable CF patients or normal subjects studied for this report.

The results of the assays of phagocytosis of selectively opsonized $P$. aeruginosa are consistent with the results of the immunofluorescence assays for receptor expression. There was little phagocytosis of unopsonized bacteria by either macrophages or neutrophils, but even low concentrations $(15 \mu \mathrm{g} / \mathrm{mL})$ of $\mathrm{IgG}$ increased the uptake of bacteria by the alveolar macrophages. Additional enhancement of phagocytosis occurred when the concentration of IgG was increased to $75 \mu \mathrm{g} / \mathrm{mL}$, but no further enhancement occurred with the highest concentration, $150 \mu \mathrm{g} /$ $\mathrm{mL}$. These results are consistent with saturation of high affinity Fc $\gamma$ R. Normal blood neutrophils, which do not express Fc $\gamma$ RI (26), did not show saturation. With alveolar macrophages, the addition of complement caused no further enhancement of phagocytosis above the level due to antibody alone. In contrast, serum treatment of antibody coated bacteria increased phagocytosis by the neutrophils very dramatically, to levels much higher than achieved by the macrophages under any condition. The observations that the effects of serum with the neutrophils were abolished by heat inactivation or $10 \mathrm{mM}$ EDTA strongly suggest that they were, in fact, due to complement fixation onto the bacteria. The fact that complement deposition on the bacteria so dramatically increased phagocytosis by the chemoattractantstimulated neutrophils while having essentially no effect with the alveolar macrophages is consistent with the expression of CR 1 and CR3 on the former but not on the latter. The lack of complement-mediated effects with the alveolar macrophages, which do express p150/95 (Leu M5), suggests that this molecule does not mediate complement dependent phagocytosis under these conditions. Thus, although this molecule is capable of promoting complement dependent adherence under some conditions in vitro (4), its true functional role in vivo and its identity as "CR4" remains to be defined.

The results with cystic fibrosis alveolar macrophages are quite similar to normal alveolar macrophages in both the receptor expression and phagocytosis assays. The pattern of HLA-DR expression on the CF cells demonstrates the power of the flow cytometer to selectively analyze the macrophages in these samples, which contained up to $70 \%$ neutrophils, since the latter do not express these class II histocompatibility antigens (23). Consistent with previous results on receptor expression $(8,9)$, fresh BAL neutrophils from the CF patients did not show as much complement-mediated enhancement of phagocytosis as did blood neutrophils, emphasizing the functional importance of the destruction of CR 1 mediated by elastase in vivo $(8,9)$.

These results allow assessment of the relative importance of $\mathrm{IgG}$ and C3-derived opsonins in vivo, and explain some previously reported immunologic abnormalities in CF. Electron microscopy studies by Thomassen et al. (27) had shown that CF BAL macrophages were quiescent and did not appear actively involved in phagocytosis. Subsequently, it has been shown that both CF sera and lung fluids contain poorly functional antibodies against $P$. aeruginosa that can actually inhibit or "block" phagocytosis (10-15). Curiously, this "blocking activity" is most notable in assays employing macrophages rather than neutrophils (11-14). Our results, which suggest that macrophages are more dependent on IgG and Fc $\gamma \mathrm{R}$ for phagocytosis than are neutrophils, suggest an explanation for those observations. Because Fc $\gamma$ RI shows more selectivity for human IgG of subclasses 1 and 3 than do the lower affinity Fc $\gamma$ RII and III (28), interference with binding of these subclasses to the bacteria is likely to result in decreased phagocytosis by macrophages. This may occur in the lung in CF because of an excess of antibodies of other subclasses $(13,14)$, and/or because of binding to the bacteria of antibodies with degraded or otherwise altered Fc $\gamma$ domains (15). Further studies using monoclonal antireceptor antibodies and/or complexes of antigen with antibodies of defined isotypes will be necessary to define more completely the role of each Fc $\gamma \mathrm{R}$ and IgG subclass in promoting Pseudomonas phagocytosis.

When considered in the context of the chronic pulmonary infection in $\mathrm{CF}$, in which neutrophils predominate, our observations that the maximal phagocytic activity of BAL macrophages and CF BAL neutrophils is so much lower than blood neutrophils are especially meaningful. Because the phagocytic activity of neutrophils is so dependent on complement, these results emphasize the importance of the complement receptoropsonin mismatch caused by elastase in the CF lung $(8,9)$. Thus, these analyses should set the stage for investigations of the potential efficacy of immunologic strategies aimed at improving the subclass distribution and function of the $\mathrm{IgG}$ in the lung in $\mathrm{CF}$ to take advantage of macrophage Fc $\gamma$ receptors, and of the use of protease inhibitors to restore the proper functioning of the complement receptors on neutrophils and their specific opsonic ligands.

Acknowledgments. The authors thank Kathleen Hilliard and Christine Wenger Tobias for administrative and technical support in preparing the manuscript.

\section{REFERENCES}

1. Unkeless JC 1989 Function and heterogeneity of human Fc receptors for immunoglobulin G. J Clin Invest 83:355-361

2. Miller LJ, Bainton DF, Borregard N, Springer TA 1987 Stimulated mobilization of monocyte Mac- 1 and p150,95 adhesion proteins from an intracellular vesicular compartment to the cell surface. J Clin Invest 80:535-544

3. Freyer DR, Morganroth ML, Rogers CE, Arnaout MA, Todd RF 1988 Modulation of surface CD11/CD 18 glycoproteins by human mononuclear phagocytes. Clin Immunol Immunopathol 46:272-283

4. Myones BL, Dalzell JG, Hogg N, Ross GD 1988 Neutrophil and monocyte cell surface p1 50,95 has iC3b-receptor (CR4) activity resembling CR3. J Clin Invest 82:640-651

5. Kindt GC, Van De Winkel J, Moore SA, Andersone CL 1991 Identification and structural characterization of Fc $\gamma$-receptors on pulmonary alveolar macrophages. Am J Physiol 260:L403-L411

6. Wahl SM, Allen JB, Welch GR, Wong HL 1992 Transforming growth factor$\beta$ in synovial fluids modulates Fc $\gamma$ RIII (CD16) expression on mononuclear phagocytes. J Immunol 148:485-490 
7. Berger M 1991 Inflammation in the lung in cystic fibrosis: a vicious cycle that does more harm than good. Clin Rev Allergy 9:119-142

8. Berger M, Sorensen RU, Tosi MF, Dearborn DG, Döring G 1989 Complement receptor expression on neutrophils at an inflammatory site, the pseudomonas-infected lung in cystic fibrosis. J Clin Invest 84:1302-1313

9. Tosi MF, Zakem H, Berger M 1990 Neutrophil elastase cleaves C3bi on opsonized pseudomonas as well as CR I on neutrophils to create a functionally important receptor mismatch. J Clin Invest 86:300-308

10. Biggar WD, Holmes B, Good RA 1971 Opsonic defect in patients with cystic fibrosis of the pancreas. Proc Natl Acad Sci 68:1716-1719

11. Thomassen MJ, Boxerbaum B, Demko CA, Kuchenbrod PJ, Dearborn DG, Wood RE 1979 Inhibitory effect of cystic fibrosis serum on pseudomonas phagocytosis by rabbit and human alveolar macrophages. Pediatr Res 13:1085-1088

12. Fick Jr RB, Naegel GP, Matthay RA, Reynolds HY 1981 Cystic fibrosis pseudomonas opsonins-inhibitory nature in an in vitro phagocytic assay. $\mathrm{J}$ Clin Invest 68:899-914

13. Eichler I, Joris L, Hsu YP, Wye JV, Bram R, Moss R 1989 Nonopsonic antibodies in cystic fibrosis. Pseudomonas aeruginosa lipopolysaccharidespecific immunoglobulin $G$ antibodies from infected patient sera inhibit neutrophil oxidative responses. J Clin Invest 84:1794-1804

14. Hornick DB, Fick Jr RB 1990 The immunoglobulin G subclass composition of immune complexes in cystic fibrosis: implications for the pathogenesis of the pseudomonas lung lesion. J Clin Invest 86:1285-1292

15. Fick Jr RB, Nagel GP, Squier SU, Wood RE, Gee JBL, Reynolds HY 1984 Proteins of the cystic fibrosis respiratory tract: fragmented IgG opsonic antibody causing defective opsonophagocytosis. J Clin Invest 74:236-248

16. Inada S, Brown EJ, Gaither TA, Hammer CH, Takahashi T, Frank MM 1983 C3d receptors are expressed on human monocytes after in vitro cultivation. Proc Natl Acad Sci 80:2351-2355

17. Simms HH, Gaither TA, Fries LF, Frank MM 1991 Monokines released during short term Fc $\gamma$ receptor phagocytosis up-regulate polymorphonuclear leukocyte and monocyte phagocyte function. J Immunol 147:265-272
18. Berger M, O'Shea J, Cross AS, Folks TM, Chused TL, Brown EJ, Frank MM 1984 Human neutrophils increase expression of $\mathrm{C} 3 \mathrm{bi}$ as well as $\mathrm{C} 3 \mathrm{~b}$ receptors upon activation. J Clin Invest 74:1566-1571

19. Sveum RJ, Chused TM, Frank MM, Brown EJ 1986 A quantitative fluorescent method for measurement of bacterial adherence and phagocytosis. J Immunol Methods 90:257-264

20. Schreiber JR, Patawaran M, Tosi MF, Lennon J, Pier GB 1990 Anti-idiotype induced, LPS-specific antibody response to $P$. aeruginosa. J Immunol 144:1023-1029

21. Schreiber JR, Pier GB, Grout M, Nixon K, Patawaran M 1991 Induction of opsonic antibodies to $P$. aeruginosa mucoid exopolysaccharide by an antiidiotypic monoclonal antibody. J Infect Dis 164:507-514

22. Berger M, Medof ME 1987 Increased expression of complement decay-accelerating factor during activation of human neutrophils. J Clin Invest 79:2 14220

23. Lampson LA, Levy R 1980 Two populations of la-like molecules on a human B-cell line. J Immunol 125:293-299

24. Lanier LL, Arnaout MA, Schwarting R, Warner NL, Ross GD 1985 p150/95, third member of the LFA-1/CR3 polypeptide family identified by anti-Leu M5 monoclonal antibody. Eur J Immunol 15:713-718

25. Tosi M, Berger M 1988 Functional differences between the $40 \mathrm{kDa}$ and 50 to $70 \mathrm{kDa}$ IgG Fc receptors on human neutrophils revealed by elastase treatment and antireceptor antibodies. J Immunol 141:2097-2103

26. Guyre PM, Campbell AS, Kniffin WD, Fanger MW 1990 Monocytes and polymorphonuclear neutrophils of patients with streptococcal pharyngitis express increased numbers of type I IgG Fc receptors. J Clin Invest 86:18921896

27. Thomassen MJ, Demko CA, Wood RE, Tandler B, Dearborn DG, Boxerbaum B, Kuchenbrod PJ 1980 Ultrastructure and function of alveolar macrophages from cystic fibrosis patients. Pediatr Res 14:715-721

28. Guyre PM, Graziano RF, Vance BA, Morganelli PM, Fanger MW 1989 Monoclonal antibodies that bind to distinct epitopes on Fc $\gamma \mathrm{RI}$ are able to trigger receptor function. J Immunol 143:1650-1655 